\title{
Charge Ordering in a Pure Spin Model: Dipolar Spin Ice
}

\author{
R. A. Borzi, ${ }^{1}$ D. Slobinsky, ${ }^{2}$ and S. A. Grigera ${ }^{2,3}$ \\ ${ }^{1}$ Instituto de Investigaciones Fisicoquímicas Teóricas y Aplicadas UNLP-CONICET and Departamento de Física, \\ Facultad de Ciencias Exactas, Universidad Nacional de La Plata, 1900 La Plata, Argentina \\ ${ }^{2}$ Instituto de Física de Líquidos y Sistemas Biológicos, UNLP-CONICET, La Plata 1900, Argentina \\ ${ }^{3}$ School of Physics and Astronomy, University of St. Andrews, St. Andrews KY16 9SS, United Kingdom
}

(Received 31 July 2013; published 2 October 2013)

\begin{abstract}
We study the dipolar spin-ice model at fixed density of single excitations, $\rho$, using a Monte Carlo algorithm where processes of creation and annihilation of such excitations are banned. In the limit of $\rho$ going to zero, this model coincides with the usual dipolar spin-ice model at low temperatures, with the additional advantage that a negligible number of monopoles allows for equilibration even at the lowest temperatures. Thus, the transition to the ordered fundamental state found by Melko, den Hertog, and Gingras in 2001 is reached using simple local spin flip dynamics. As the density is increased, the monopolar nature of the excitations becomes apparent: the system shows a rich $\rho$ vs $T$ phase diagram with "charge" ordering transitions analogous to that observed for Coulomb charges in lattices. A further layer of complexity is revealed by the existence of order both within the charges and their associated vacuum, which can only be described in terms of spins-the true microscopic degrees of freedom of the system.
\end{abstract}

DOI: 10.1103/PhysRevLett.111.147204

PACS numbers: 75.10.Hk, 02.70.Uu, 75.50.-y

Defects are the entropic antagonists of the perfectly ordered state, while at the same time they are inextricably linked to it [1]. Topological defects are particularly stable forms of disorder which, when dense, can lead to higher hierarchies of order. Thus, for example, the interaction between vortices in a superconductor can form an Abrikosov lattice or other forms of vortex matter [2]; these phases, in turn, will have their own topological defects. The Berezinskii-Kosterlitz-Thouless transition (BKT), dealing with the unbinding of vortex-antivortex pairs $[3,4]$ is another example. Among the endless variety of these defects [5], a new kind of fractional pointlike topological excitation-magnetic charges or monopoles - was proposed theoretically [6] and tested experimentally [7] in the spin-ice compounds. Given their analogy with electrical charges [6,8], a rich behavior is to be expected at low temperature $(T)$ for high monopole number density $(\rho)$. These are two conditions that are very difficult to achieve simultaneously in spin ice, and require a very fine tuning of the parameters of the Hamiltonian. In this work we take the alternative path of studying the full $\rho$-T phase diagram in a spin ice system by externally fixing the density of magnetic monopoles.

Controlling the density of topological defects is a clean way of highlighting their essential role in determining some ordered phases. During the 1980s and 1990s, the importance of vortex strings in the 3D $X Y$ model and of "hedgehog" point defects in the Heisenberg model phase transition was clearly shown using this strategy $[9,10]$. We take a similar approach in this Letter: we fix the number of defects, but keep the model unbiased and simulate the spinice system not in terms of the effective degrees of freedom (the monopoles), but in terms of the individual spins.
The strength and beauty of the monopolar picture of this magnetic system appears reinforced by our finding of two phases, which can be understood in terms of the different types of ordering of the attracting monopoles (i.e., chargelike degrees of freedom). Adding to this remarkable result, our perspective shows in a unified view the presence of more subtle forms of order: they are related to the many different ways in which both the monopole-free system (the monopole vacuum) and a perfect crystal of single monopoles can be assembled in terms of their constituent spins. These spin degrees of freedom that are not taken into account in the monopolar picture can be thought in this context as internal degrees of freedom of the monopolar charges and vacuum. The close relationship between these findings and previous results in spin ice is discussed.

The magnetic properties of spin-ice (SI) materials at low temperatures are well described by the dipolar model $[11,12]$, in which nearest neighbors exchange, $J$, and long ranged dipolar interactions with coupling constant $D$-both measured in Kelvin-are taken into account in the Hamiltonian

$$
\begin{aligned}
\frac{\mathcal{H}}{T}= & \frac{D}{T}\left(\frac{J}{3 D} \sum_{<i j>} S_{i} S_{j}\right. \\
& \left.+a^{3} \sum_{(i, j)}\left[\frac{\hat{e}_{i} \cdot \hat{e}_{j}}{\left|\mathbf{r}_{i j}\right|^{3}}-\frac{3\left(\hat{e}_{i} \cdot \mathbf{r}_{i j}\right)\left(\hat{e}_{j} \cdot \mathbf{r}_{i j}\right)}{\left|\mathbf{r}_{i j}\right|^{5}}\right] S_{i} S_{j}\right) .
\end{aligned}
$$

Here, the magnetic moments $\left(\boldsymbol{\mu}_{i}\right)$ occupy the sites $i$ of a pyrochlore lattice, separated by distances $\left|\mathbf{r}_{i j}\right|$. They reside in the vertices of corner-shared tetrahedra (see Fig. 1) of side $a$ and behave as Ising-like spins $\left(\boldsymbol{\mu}_{i}=\mu S_{i} \hat{e}_{i}\right.$, with $S_{i}= \pm 1$ ), constrained to point along the $\langle 111\rangle$ directions $\hat{e}_{i}$. When the effective nearest neighbors interaction 


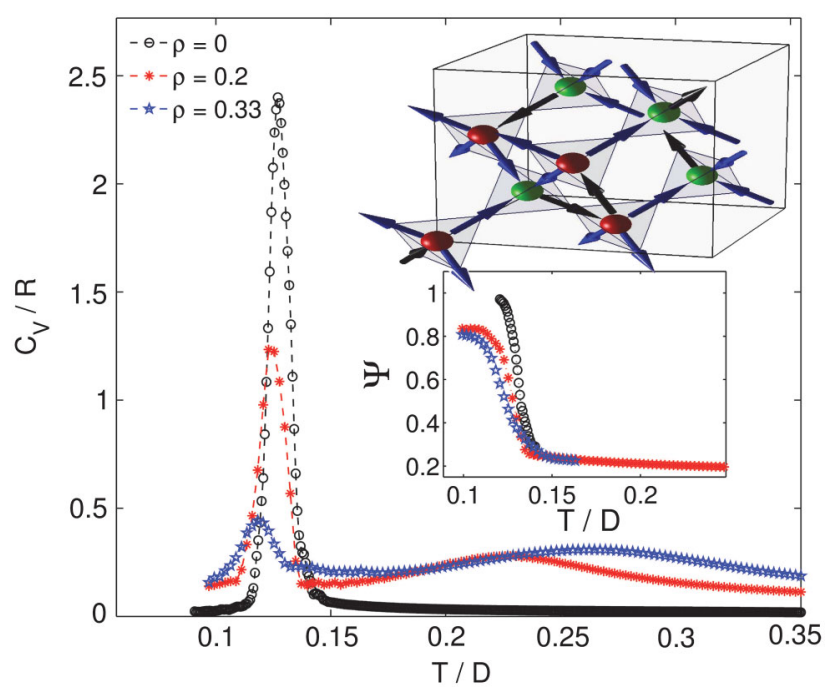

FIG. 1 (color online). Molar specific heat $C_{V}$ as a function of $T$ for three different monopole densities $(\rho)$. The peak in $C_{V}$ at $T_{V}$ marks the first order phase transition to an ordered spin phase. The condensation of the monopoles into a magnetic crystal is signaled by a broader peak at a higher temperature $T_{C}$. Top inset: Cubic unit cell for spin-ice materials. The Ising-type spins (shown as black arrows for in and blue for out of "up" tetrahedra) illustrate one of the ground state configurations found for the monopole crystal. Both types of single monopoles are plotted as colored balls, which conform an "ionic" crystal. Lower inset: Temperature dependence of the order parameter for the spin-ice ground state [17].

$J_{\text {eff }}=J / 3+5 / 3 D>0, D=\mu_{0} \mu^{2} / 4 \pi a^{3}$, the spin-ice rule is enforced: two spins should point in and two out of a tetrahedron to minimize its energy. This rule, combined with the lattice geometry makes SI a magnetic analogue of water ice, with a similar residual entropy [11]. A violation of the local law implies the creation of a defect, or monopole, sitting in the tetrahedron with a magnetic charge proportional to the divergence of the spin vectors [6]. The number of defects at fixed temperature is thus regulated by the magnitude of $J_{\text {eff }} / D$. In the currently known SI materials, it leads to moderately correlated monopole fluids $[13,14]$. Material design or the application of external pressure can be used to strengthen the correlations, revealing new aspects of these systems that we set out to determine by numerical simulations.

Here we have used the Monte Carlo technique to simulate the dipolar SI model [Eq. (1)] with Ewald summations. We modified the dynamics so that we can have independent control over the temperature, measured in units of $D$, and over the density of magnetic charges. Starting from a random configuration satisfying the ice rules, we first flip enough spins to reach the desired number of positive and negative single excitations per tetrahedra without allowing any double charge excitation. We then follow the usual single flip Metropolis algorithm with an additional constraint: we forbid spin flips which either create or destroy single defects, preserving detailed balance. In other words, we work in a statistical ensemble with a constant number of single monopole defects, instead of fixed chemical potential [15]. Other details of the simulations can be found in the Supplemental Material [16].

The curves in Fig. 1 represent our results for the molar specific heat $C_{V}$ as a function of temperature at low $\rho$ and linear lattice size $L=3$ unit cells. The limit $\rho \ll 1$ is particularly important, since at very low $T$ we expect our monopole conserving model to coincide with the usual dipolar SI model. $\rho=0$ is taken as the minimum nontrivial number of monopoles (two, of opposite sign). At this concentration, the evolution of the system consists in the exploration of the states belonging to an almost perfect SI manifold (the exponentially degenerate set of two-in-twoout states) by means of the random wanderings of the two defects. Although no phase transition is expected in terms of charges, the specific heat in Fig. 1 shows a sharp peak for $\rho=0$, centered at $T_{V} \approx 0.13 D$. An identical feature in $C_{V}$ was found by R. Melko and collaborators in the usual SI dipolar model using a multiple spin flip "loop" algorithm [17]. They identified the peak at $T_{V}$ with a first order transition to a SI ordered ground state, and proposed an order parameter $(\Psi)$ to account for this order [17]. In the present context, this ordering in the spin system with a virtual absence of monopoles should be interpreted as a change of the internal state of the vacuum of magnetic charges. The lower inset to Fig. 1 shows how $\Psi$ grows below $T_{V}$ in our simulations. The quantitative agreement [12] — which holds also for other quantities — confirms that this minimum number of conserved defects is sufficient to allow equilibration, even when the evolution is simulated through a simple single spin-flip algorithm. This result suggests that the extreme paucity in monopole excitations at low temperatures is a major factor in the spin freezing observed experimentally.

An inspection of Fig. 1 and its lower inset shows thatalbeit affected by difficulties in equilibrating and finite size effects - the vacuum of charges also orders below $T_{V}$ for non-negligible $\rho$. More interestingly, these concentrations show a second (wider) maximum in $C_{V}$, occurring at a temperature $T_{C}(\rho)$ higher than $T_{V}$. Both $T_{C}(\rho)$ and the height of the peak tend to increase with $\rho$, hinting at a connection with the onset of ordering of the magnetic charges. We expect the Coulomb attraction to favor the clustering of monopoles in the zinc blende structure, where one type of monopole has a greater tendency to occupy either the up or down tretahedra sublattices. In analogy with the staggered magnetization for antiferromagnetism, we use the staggered charge density, $\left|\rho_{S}(T)\right|$, to quantify this type of alternating or staggered charge order (SCO). We define $\left|\rho_{S}(T)\right|$ as the average of the modulus of the total magnetic charge in up tetrahedra per sublattice site per unit charge. Figure 2 shows $\left|\rho_{S}(T)\right|$ as a function of temperature for different sizes $L$ and fixed density $\rho=0.1$. We can 


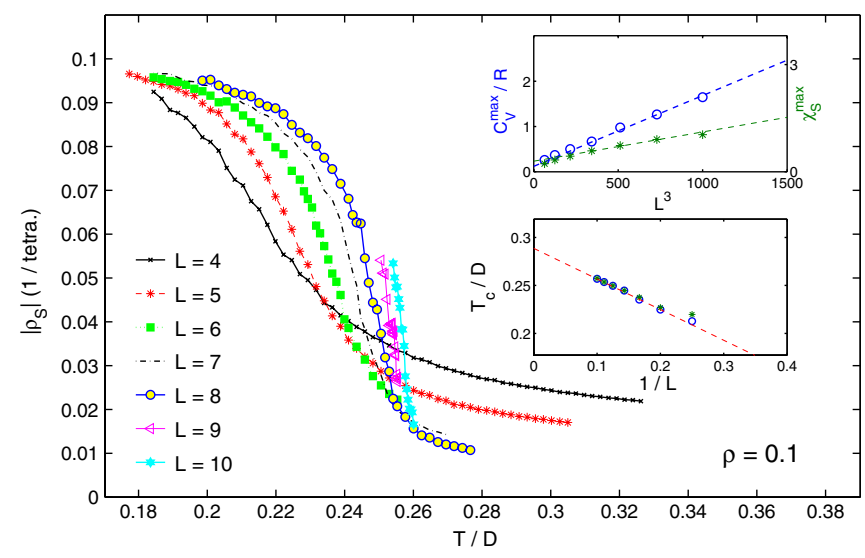

FIG. 2 (color online). Average modulus of the staggered density $\left|\rho_{S}\right|$ as a function of $T$, for $\rho=0.1$. We focus near the crystallization transition. On increasing the system size $L$, the order parameter decreases more abruptly near the transition temperature $T_{C}(L)$. The fluctuations in energy (blue trace with open circles) and those in $\left|\rho_{S}\right|$ (green asterisks) increase with $L^{3}$ (top inset) and $T_{C}$ evolves with $L^{-1}$ (lower inset), as in a first order transition.

see that $\left|\rho_{S}(T)\right|$ tends to $\rho$ at low temperatures, decreasing to a small but finite value at high temperatures that scales as $L^{-3 / 2}$, as expected (see Supplemental Material [16]). The transition becomes sharper with $L$, while the temperature of its steepest slope increases. The top inset to Fig. 2 shows the fluctuations in energy $\left(C_{V}\right)$ and in $\left|\rho_{S}(T)\right|\left(\chi_{S}\right)$ measured at the temperature $T_{C}(L)$ at which they peak. Both tend to increase proportional to the volume of the system, while $T_{C}(L)$ vs $1 / L$ displays a linear behaviour (lower inset), indicating a first-order phase transition [18]. We have also measured histograms for the local monopole number density, $\rho_{\text {loc }}$, which become bimodal below $T_{C}$, with peaks near $\rho_{\text {loc }}=1$ and $\rho_{\text {loc }}=0$. This signals that in addition to developing a staggered ordering with a net charge in each sublattice, the system phase separates into a dense arrangement of monopoles (an ionic crystal of magnetic charges) and a "fluid" phase with a very low local concentration of monopoles.

The top part of Fig. 3 shows $C_{V}$ as a function of temperature for a wide range of $\rho$, and $L=4$. A small peak is still noticeable at $T_{V}$, even for high $\rho$. The height of the cusp at $T_{C}$ grows with $\rho$ for small concentrations, as a consequence of the increase in the relative fraction of crystalline phase being formed. At $\rho=0.3$ the cusp becomes wider, and eventually resolves into two well-defined peaks for $\rho \geq$ 0.5. This peak bifurcation corresponds to the decoupling between the onset of the staggered order and monopole crystallization. The low temperature peak is linked with the second phenomenon, and has a very modest evolution with $\rho$, occurring always at a temperature (which we call $T_{C}$ ) below $\approx 0.3 D$. The opposite is true for the second cusp, that peaks at temperature $T_{S}$ going above $0.7 D$ for $\rho>0.6$. To show this explicitly, we plot $\rho_{S}(T)$ in the bottom part of

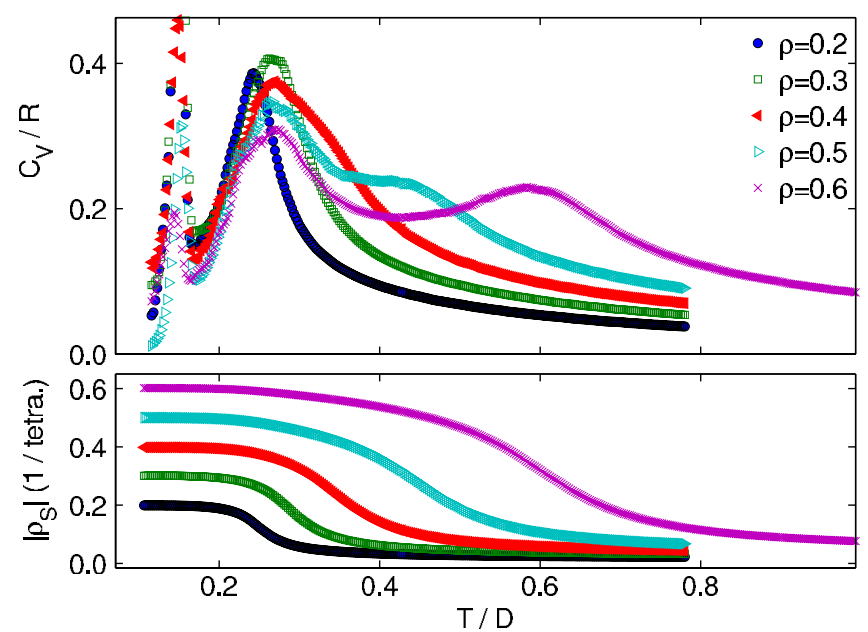

FIG. 3 (color online). Upper panel. Molar specific heat $\left(C_{V}\right)$ as a function of $T$ for $L=4$, at densities indicated in the legend. At very low temperatures, a peak at $T_{V}$ indicates the ordering of the vacuum even at high $\rho$. A broader peak at a higher temperature, $T_{C}$, signals a transition in the charge degrees of freedom. For $\rho>0.3$ this transition splits into two: one at $T_{C}$ and a second one at $T_{S}$. Lower panel. Staggered density $\rho_{S}(T)$ for the same densities. For $\rho>0.3$, the onset of SCO coincides with the high temperature peak in $C_{V}$.

Fig. 3, for the same values of $\rho$ as the upper panel. We observe that while for small $\rho$ the temperature at which $\rho_{S}$ is steepest correlates with $T_{C}$, it clearly follows the behavior of $T_{S}$ for $\rho>0.3$. In other words, the position of the high temperature peak, $T_{S}$, marks the transition to a phase with long-range staggered charge ordering, with no phase coexistence. It is only below $T_{C}$ that a magnetic crystal separates from a very low vapor pressure fluid. Between $T_{S}$ and $T_{C}$ the average local density is homogeneous, fluidlike, but with a finite tendency $\rho_{S}$ for positive and negative charges to occupy preferentially separate sublattices.

We summarize most of the results found on this work in Fig. 4. It shows, projected into the $\rho$ vs $T$ plane, the phase diagram for our model, drawn on top of an interpolated contour plot of the specific heat data. The low temperature dome we have labeled as $T_{C}(\rho)$ corresponds to the first order transition below which a monopole crystal with staggered order coexists with a low vapor-pressure gas [19]; see also the mean field treatment in [21]. At lower temperature, $T_{V}$ indicates the spin ordering ("vacuum order") first reported in [17]. Above $T_{C}(\rho)$ and small $\rho$, the system exists in a fluid phase characterized by a homogeneous average local density of monopoles and no long range charge order. As we increase the density we reach a bifurcation point near $\rho=0.3$ where the crystallization transition splits in two: $T_{S}(\rho)$ marks the onset of SCO with homogeneous local density, while the system phase separates at $T_{C}(\rho)$ [22].

Figure 4 has a startlingly similar counterpart in systems of simple electric charges in a lattice, where $T_{S}$ was 


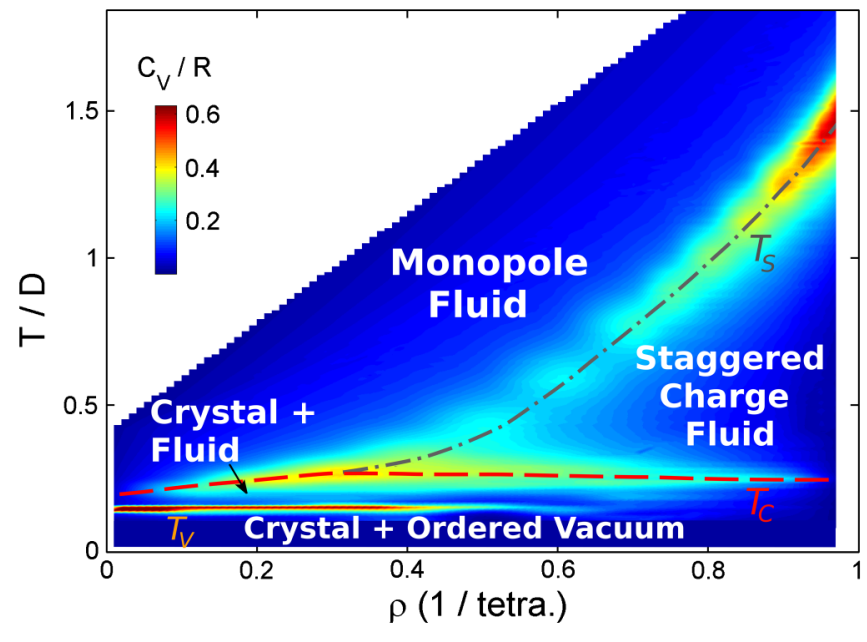

FIG. 4 (color online). $\quad T$ vs $\rho$ phase diagram for the dipolar spin ice. At high temperatures, the system exists in a fluid phase of monopoles with no long range order. As $T$ is lowered at low $\rho$ the fluid undergoes a first order phase transition at $T_{C}(\rho)$, and crystallises into the zinc blende structure. At higher densities, the crystallization develops in two stages: the onset of staggered order at $T_{S}$ and phase separation at $T_{C}$. A tricritical point at $\rho \approx 0.3$ and $T / D \approx 0.3$ marks the meeting point of $T_{C}$ and $T_{S}$. Below $T_{V}$, the spin degrees of freedom of the vacuum undergo an ordering transition. The diagram is overlaid on top of an interpolated contour plot of $C_{V}$ for $L=4$ (see Fig. 3).

identified as a second order Néel-like transition temperature, meeting at a tricritical point with a first order dome where an ionic crystal coexists with a low density disordered phase $[21,24,25]$. A finite size scaling analysis of our results confirms that $T_{S}$ is a second order transition within the 3D-Ising universality class (see Supplemental Material [16]). Beyond these similarities, one fundamental difference is that in our case the true degrees of freedom are spins, and the presence of the charges-and their ordering-are emergent phenomena.

A broader view is gained by comparison with other spin systems. As in our case, the melting of the ionic crystal in the uniformly-frustrated $X Y$ spin model in the triangular lattice can take place in two stages. However, this is a twodimensional system with a continuous symmetry and therefore one of these transitions is of a different character (BKT) [26]. The resemblance to our system is closer in the case of the Blume-Emery-Griffiths Model [1], and it extends even to the universality class expected for the tricritical point.

The approximate character of the monopole picture implies that there can be order not only in the internal structure of the monopoles vacuum, but also within the magnetic charges themselves [27]. Indeed, we checked that different spin configurations leading to the same monopole cluster can have different energies, and found a good candidate for the ground state of the magnetic crystal, by exploring all possible spin configurations within the conventional cubic unit cell that would generate a perfect zinc blende structure. Among the 48 configurations satisfying this condition, 16 had minimum energy, differing from the next low lying energy level by about $6 \%$. As illustrated in the top inset to Fig. 1, the symmetry-connected ground states are characterized by having zero magnetization, with the magnetic moments of each of the four up tetrahedra pointing along the four possible $\langle 111\rangle$ diagonals.

Our $\rho$-T phase diagram in Fig. 4 can be related to that measured in $\mathrm{Dy}_{2} \mathrm{Ti}_{2} \mathrm{O}_{7}$ and $\mathrm{Ho}_{2} \mathrm{Ti}_{2} \mathrm{O}_{7}$ under a field $\mathbf{H} / /[111][6,28-30]$. A field in this direction induces a polarized state with monopoles in a crystalline zinc blende structure and $\rho=1$ [6]. At low temperatures this phase is accessed through a first order metamagnetic phase transition for fields of the order of 1 tesla. This curve of metamagnetic transitions in the $H-T$ phase diagram corresponds to the dome of first order phase transitions in the $\rho$-T phase diagram, $T_{C}$, in the same way that the first order transition line in the pressure-temperature diagram for a vapor-liquid transition becomes a region in the temperature-density plane. The tricritical point in the $\rho-T$ diagram corresponds to the critical point of the first order transition line in $H-T$ and the line of second order transitions $T_{S}$ marking spontaneous symmetry breaking is replaced by a line of crossovers in the $H-T$ plane, since $H$ is a symmetry breaking field $[6,28,30]$.

In summary, using the dipolar SI model we have been able to explore the whole $\rho$ vs $T$ phase diagram, observing chargelike ordering in a purely magnetic system. Our model excludes the possibility of double charges, a fact that limits its application to all possible SI materials and temperatures. However, it has served to explore a complex phase diagram, stressing in a unified view both the monopolar nature of the excitations and the unavoidable need to take into account their spin nature at very low temperatures.

We thank R. Moessner, C. Castelnovo, T. S. Grigera, D. Cabra and G. Rossini for helpful discussions, and the financial support of CONICET and ANPCYT (Argentina), and the Royal Society (UK).

[1] P. Chaikin and T. Lubensky, Principles of Condensed Matter Physics (Cambridge University Press, Cambridge, England, 2000).

[2] G. Blatter, V. B. Geshkenbein, A. I. Larkin, and V.M. Vinokur, Rev. Mod. Phys. 66, 1125 (1994).

[3] V. Berezinskii, Sov. Phys. JETP 32, 2 (1971).

[4] J. Kosterlitz and D. Thouless, J. Phys. C 6, 1181 (1973).

[5] N. D. Mermin, Rev. Mod. Phys. 51, 591 (1979).

[6] C. Castelnovo, R. Moessner, and S. Sondhi, Nature (London) 451, 42 (2008).

[7] D. J. P. Morris et al., Science 326, 411 (2009).

[8] S. R. Giblin, S. T. Bramwell, P. C. W. Holdsworth, D. Prabhakaran, and I. Terry, Nat. Phys. 7, 252 (2011).

[9] G. Kohring, R. E. Shrock, and P. Wills, Phys. Rev. Lett. 57, 1358 (1986). 
[10] M.-H. Lau and C. Dasgupta, Phys. Rev. B 39, 7212 (1989).

[11] S. Bramwell and M. Gingras, Science 294, 1495 (2001).

[12] R. G. Melko and M. J. Gingras, J. Phys. Condens. Matter 16, R1277 (2004).

[13] H. D. Zhou et al., Nat. Commun. 2, 478 (2011).

[14] H. D. Zhou, J. G. Cheng, A. M. Hallas, C. R. Wiebe, G. Li, L. Balicas, J.S. Zhou, J. B. Goodenough, J. S. Gardner, and E. S. Choi, Phys. Rev. Lett. 108, 207206 (2012).

[15] L. D. C. Jaubert and P. C. W. Holdsworth, Nat. Phys. 5, 258 (2009).

[16] See Supplemental Material at http://link.aps.org/ supplemental/10.1103/PhysRevLett.111.147204 for a brief description of the simulations, methods, finite size scaling and other details..

[17] R. Melko, B. den Hertog, and M. Gingras, Phys. Rev. Lett. 87, 1 (2001).

[18] K. Binder and D. W. Heermann, Monte Carlo Simulation in Statistical Physics (Springer, Berlin, 1997).

[19] Though not shown in Fig. 4, we expect $T_{C} \rightarrow 0$ in the limit $L^{3} \rightarrow \infty, \rho \rightarrow 0$ (as in a binary mixture with attractive interactions between like atoms stronger than between unlike atoms [20]).
[20] C. Kittel and H. Kroemer, Thermal Physics (W.H. Freeman, New York 1980).

[21] R. Dickman, AIP Conf. Proc. 492, 225 (1999).

[22] This double transition is missing in the phase diagram of Ref. [23] (Fig. 5), given the absence of a lattice in their Debye-Huckel model.

[23] C. Castelnovo, R. Moessner, and S. Sondhi, Annu. Rev. Condens. Matter Phys. 3, 35 (2012).

[24] R. Dickman, Braz. J. Phys. 30, 711 (2000).

[25] A. Ciach and G. Stell, Phys. Rev. Lett. 91, 1 (2003).

[26] S. E. Korshunov and G. V. Uimin, J. Stat. Phys. 43, 1 (1986).

[27] In the same way that there are six different forms in which a two-in-two-out state can be assembled in a given tetrahedron, there are four different ways to construct a monopole with a single charge of a given sign.

[28] T. Sakakibara, T. Tayama, Z. Hiroi, K. Matsuhira, and S. Takagi, Phys. Rev. Lett. 90, 3 (2003).

[29] R. Higashinaka, H. Fukazawa, and Y. Maeno, J. Phys. Soc. Jpn. 73, 2845 (2004).

[30] C. Krey, S. Legl, S. R. Dunsiger, M. Meven, J. S. Gardner, J. M. Roper, and C. Pfleiderer, Phys. Rev. Lett. 108, 1 (2012). 